\title{
An axiomatic analysis of ranking sets under simple categorization
}

\author{
José C. R. Alcantud · Ritxar Arlegi
}

Received: 9 November 2010 / Accepted: 16 April 2011 / Published online: 10 May 2011 (C) The Author(s) 2011. This article is published with open access at SpringerLink.com

\begin{abstract}
This paper contributes to the axiomatization of additive rules for ranking sets of objects under the psychological principle of categorization. Firstly we proceed with the case where the elements in the sets are categorized into at most three groups, namely good (with value 1), neutral (with value 0 ), and bad (with value -1 ). Secondly, we solve the case where there are only good and neutral elements. In both instances the evaluation of the sets is purely additive. Lastly, we show that dropping one of the axioms in our general characterization produces an axiomatization of the more general class of evaluations where good and bad elements are weighted differently. Areas of research in Economics such as committee selection problems, hedonic games and matching are among the ranking sets models where our results could potentially be applied.
\end{abstract}

Keywords Ranking sets · Additive representation · Categorization

JEL Classification D01 $\cdot$ D71 $\cdot$ D81

José C. R. Alcantud acknowledges financial support from the Spanish Ministerio de Ciencia e Innovación (Project ECO2009-07682), and from Junta de Castilla y León (Project SA024A08 and GR-99 Funding). R. Arlegi (corresponding author) acknowledges financial support from the Spanish Ministerio de Ciencia e Innovación (Projects SEJ2006-11510 and ECO2009-12836), and from Junta de Castilla y León (Project VA040A05). We are grateful for constructive comments and suggestions by an anonymous referee.

J. C. R. Alcantud

Facultad de Economía y Empresa, Universidad de Salamanca, 37008 Salamanca, Spain e-mail: jcr@usal.es

R. Arlegi (凶)

Department of Economics, Universidad Pública de Navarra, Campus Arrosadia, 31006 Pamplona, Navarre, Spain

e-mail: rarlegi@unavarra.es 


\section{Introduction}

Barberà, Bossert and Pattanaik devote the 17th chapter of the Handbook of Utility Theory (2nd volume) to the problem of "Ranking Sets of objects" (cf. Barberà et al. 2004). Formally, the elementary question in ranking sets models is the following: How should the possible subsets of $X$, a universe of elements, be ranked? Obviously the answer depends very much on the particular context under inspection, that is, on the interpretation of the elements of $X$, its subsets, and the binary relation that ranks them. As a matter of fact, in the aforementioned chapter the authors provide a thoughtful survey showing how ranking sets models apply in very diverse economic settings, including social choice correspondences (see, for example, Gärdenfors 1976; Kelly 1977; Pattanaik 1978), committee selection problems (Barberà et al. 1991), the study of voters' strategies under approval voting (Brams and Fishburn 1978), hedonic games (Banerjee et al. 2001; Bogomolnaia and Jackson 2002), many-to-one matching problems (Gale and Shapley 1962; Roth and Sotomayor 1990), problems of choice under complete uncertainty (Barberà et al. 1984; Bossert et al. 2000; Arlegi 2007), the approval of a number of issues or norms in a committee or legislative body (Barberà et al. 1991), the ranking of opportunity sets (Jones and Sugden 1982, Pattanaik and Xu 1990; Sugden 1998), and the measurement of the qualitative (subjective) probability of events (Kraft et al. 1959; Scott 1964).

In this paper we present several results, both at a theoretical level and as applied to different contexts, that shed light on how sets should be ranked under the assumption that the agent performs a kind of simple categorization of the elements of the initial universe of elements $X$. Simplicity in categorization is captured by the fact that the agent conceives of a reduced list of classes, namely no more than "good", "neutral" or "bad" elements. This might seem to involve a considerable loss of generality but we show that there are a sizable number of settings where such an assumption is compelling.

The are numeros reasons why an agent might perform such a rough categorization. It is sometimes the case that the agent cannot further discriminate due to a bounded perceptive capacity or to a lack of information. In other cases it is not necessary for the agent to discriminate further given the particular problem in hand. A third reason, related to both of the above, is that a more refined discrimination could produce better results at the expense of a sacrifice. Categorization is then the natural response to the need to simplify a complex decision-making problem and to economize on the cost of processing all the information. This is in fact a well-recognized principle that prevails in cognitive and social psychology, especially since Allport (1954).

For example, candidates for entry into a coalition or assembly might be desirable or not depending merely on whether they belong to the agent's party and are expected to cast their vote in favor or against his/her interests; or new candidates for a job or for membership of a club or committee might be evaluated solely according to whether they fulfill certain qualification requirement or hold a certain entitlement (see Samet and Schmeidler 2003; Dimitrov et al. 2007). Similarly, an individual who professes a certain religion or has a particular political ideology might evaluate a set of norms exclusively according to whether they are compatible with the principles of his/her religion or ideology. When evaluating the freedom of choice that an opportunity set 
provides it is sometimes the case that there is a partition between opportunities that increase the freedom of the agent and opportunities that do not (see Romero-Medina 2001). Finally, in a context of uncertainty there are situations where the feasible outcomes are evaluated depending solely on their compatibility with the agent's interests (see Bogomolnaia et al. 2005; Bogomolnaia and Moulin 2004). ${ }^{1}$

Furthermore, the relevance of the kind of simple categorization that we propose lies in the fact that it turns out to have theoretical implications, for example regarding strategy-proofness in voting (Brams and Fishburn 1978, 2002), efficiency and strategyproofness in collective choice problems in an uncertainty context (Bogomolnaia et al. 2005), the specification of group formation models (Barberà et al. 2001), stability in hedonic games (Dimitrov et al. 2006), and the economic evaluation of individual freedom of choice (Pattanaik and Xu 1990; Romero-Medina 2001).

In line with the method adopted in the ranking sets literature, we follow an axiomatic approach to the problem. We start by analyzing the consequences of three elementary axioms, and then investigate the formal implications of relaxing the initial axiomatic structure of the problem.

A basic feature of the categorization phenomenon is that it simplifies the decision problem by narrowing the range of comparative judgements of the decision maker among the basic elements. Two of the three axioms that we propose capture the kind of simplicity that we attribute to such a reduction process. On the one hand, Symmetry within categories (SWC) expresses that categorization is simple in the sense that once an item has been revealed as good, neutral or bad the agent does not discrimine further. On the other hand, Symmetry across categories (SAC) establishes that when good and bad elements exist, no category is stronger or weaker than the other. This is done by imposing that the desirability of any element that has been revealed as good (bad) must be offset by the undesirability of any element that has been labeled as bad (good). We add a requirement of separability called Independence (IN). Conditions of this type hark back to de Finetti (1931) and are widely used in ranking sets literature.

The conditions above permit to infer which are the good, neutral and bad elements endogenously from the information given by the ranking over the sets. ${ }^{2}$ Thus, our first result shows that the three axioms are fulfilled if and only if the agent ranks sets as if: (1) the three categories of elements (good, neutral and bad) were attached to values of $-1,0$, and 1 respectively; and (2) the value of the subsets were calculated by the mere addition of the valuations of their elements. We have called this the Good-Neutral-Bad rule ("GNB rule").

As for the analysis of the consequences of relaxing the axiomatic structure of the problem, the most interesting result arises when the sometimes controversial axiom SAC is dropped. In that case, the two remaining axioms (SWC and Independence) characterize a meaningful family of rankings where the agent performs a weighted

\footnotetext{
1 In many of the examples mentioned categorization is made only in a dichotomic "good-bad" way. Our characterization results include this as a particular case where no "neutral" category exists.

2 Other papers such as Jones and Sugden (1982), Pattanaik and Xu (1990) and Romero-Medina (2001) also consider some kinds of categorization among significant and non-significant elements, but such a distinction is made, ex-ante, as a primitive of the model. Moreover, they only apply to the particular problem of ranking opportunity sets.
} 
addition of the positive and negative values of the elements that have been revealed, respectively, as good and bad. We call this the "weighted GNB rule". Barberà et al. (2001), for example, use such a rule in a group formation model.

As noted above, the results also apply to situations where categorization is made in a dichotomous "good/bad" way, with no "neutral" category (as in the study of approval voting procedures by Brams and Fishburn 2002). Likewise, particular specifications include cases where there are no bad elements, such as the opportunity sets ranking problem.

In addition to the fact that they arise from our axioms, we believe that both the GNB and the weighted GNB rules are natural and interesting as such. In particular, they are conspicuous cases of the family of separable preferences over sets, which are commonly used in the analysis of voting situations (Barberà et al. 1991; Berga et al. 2004; Ju 2003, 2005) and coalition formation games (Burani and Zwicker 2003; Dimitrov et al. 2006). They are also prominent examples of additively representable rankings over sets, a special subfamily of separable preferences that also arise in many economic contexts (see, for instance, Alcantud and Arlegi 2008).

The rest of the paper is organized as follows. Section 2 contains some fundamentals of the model. Section 3 sets out the three basic axioms that characterize the GNB rule. All the characterization results are stated and proved in Sect. 4: the characterization theorem and the corresponding proof for the GNB rule are presented in Sect. 4.1. Section 4.2 explores some consequences of dropping or relaxing the SWC axiom. One of them is that a rule proposed in Alcantud and Arlegi (2008) turns out to be a special case of a GNB rule. Section 4.3 proves that by dropping SAC the aforementioned weighted GNB rule becomes characterized. Section 5 contains some final comments.

\section{Basic setup}

Hereafter $X$ represents the universal set of elements which, unless otherwise stated, is assumed to be finite. $\chi_{0}$ denotes the set of subsets of $X$, including the empty set. We denote by $\succcurlyeq$ a binary relation, defined on $\chi_{0}$, which is complete and transitive (i.e. a complete preorder or "ordering"). The asymmetric factor and symmetric factor of $\succcurlyeq$ are denoted, respectively by $\succ$ and $\sim$. In general we interpret $\succcurlyeq$ as a weak preference relation among sets, and $\succ$ and $\sim$ as the corresponding strict preference and indifference relations. $\xi$ stands for the set of all orderings on $\chi_{0}$, and $\neg$ denotes logical negation.

Definition 1 Associated with $\succcurlyeq \in \xi$, the characteristic function $\mathbf{c}$ of $(X, \succcurlyeq)$ is defined by the following expression: For each $x \in X$,

$$
\mathbf{c}(x)=\left\{\begin{array}{cl}
-1 & \text { if } \varnothing \succ\{x\} \\
0 & \text { if }\{x\} \sim \varnothing \\
1 & \text { if }\{x\} \succ \varnothing
\end{array}\right.
$$


Definition 2 The ranking of sets $\succcurlyeq$ is said to be a GNB ranking if

$$
\text { for all } A, B \in \chi_{0}: A \succcurlyeq B \text { if and only if } \sum_{a \in A} \mathbf{c}(a) \geqslant \sum_{b \in B} \mathbf{c}(b)
$$

where $\mathbf{c}$ stands for its characteristic function and $\sum_{a \in \varnothing} \mathbf{c}(a)$ is defined as 0 .

The interpretation of a GNB rule is plain: a ranking is GNB-type when it is additive on the basis of its characteristic function.

The characteristic function, $\mathbf{c}$, of $(X, \succcurlyeq)$ is a particular case of what we call a $G N B$ function, namely, any function $c: X \longrightarrow\{-1,0,1\}$, which can be interpreted as if the agent had classified the elements of $X$ into three categories, interpreted as Good (label 1), Neutral (label 0), and Bad (label -1). Therefore, Definition 2 includes the case where there exists a GNB function $c$ such that for all $A, B \in \chi_{0}: A \succcurlyeq B$ if and only if $\sum_{a \in A} c(a) \geqslant \sum_{b \in B} c(b)$ with the convention $\sum_{a \in \varnothing} c(a){ }^{3}$ This in turn contains the instance where $c: X \longrightarrow\{0,1\}$, which is the subject of specific analysis in Alcantud and Arlegi (2008, Section 4). It is worth mentioning that the latter setting incorporates two special subcases. On the one hand, if $c(x)=1$ for all $x \in X$, the cardinality rule that Pattanaik and Xu (1990) propose in the context of ranking opportunity sets in terms of freedom of choice is obtained. On the other hand, when $c(x)=0$ for all $x \in X$ the corresponding GNB rule is trivial: the universal indifference rule (according to which $A \sim B$ for all $A, B \in \chi_{0}$ ) appears.

Since any binary relation that arises from (1) is necessarily a complete preorder, no insight is lost by restricting the study to this class of relations.

The model for GNB rules (and its extension in Sect. 4.3 below) finds a formal intimation to the proposal in Fishburn (1992). Nevertheless, differences in the approaches are noticeable. Our final Sect. 5 explores this issue in more detail. At this point we emphasize that our objective is to propose axiomatic characterizations of various rules, and that such characterizations are made under the assumption of simple categorization. This is not the case of Fishburn (1992) insightful analysis of general properties of self-reflecting signed orders and conditions for their extension to subset preferences in more general contexts.

\section{Ranking sets under simple categorization: axioms}

In this section we present and discuss three simple axioms that seek to capture the key properties that we associate with the phenomenon of simple categorization. In Sect. 4 below we prove that these three axioms characterize the family of orderings on $\chi_{0}$ that are additively representable on the basis of its characteristic function (cf. Theorem 4.1). Subsequently, we explore the possibilities and consequences of relaxing or dropping some of the axioms.

\footnotetext{
3 An important reason for taking the characteristic function $\mathbf{c}$ as a primitive of the model instead of $c$ is that it enables the partition among good, neutral and bad elements to be obtained endogenously from the information given by the ranking $\succcurlyeq$.
} 
- Symmetry within categories (SWC). If $(\{a\} \succ \varnothing$ and $\{b\} \succ \varnothing)$ or $(\varnothing \succ\{a\}$ and $\varnothing \succ\{b\})$ then $\{a\} \sim\{b\}$.

- Symmetry across categories (SAC). If $\{a\} \succ \varnothing \succ\{b\}$ then $\{a, b\} \sim \varnothing$.

- Independence (IN). $\forall A, B, C, D \in \chi_{0}$ such that $(A \cup B) \cap(C \cup D)=\varnothing$ and $C \sim D, A \succcurlyeq B$ if and only if $A \cup C \succcurlyeq B \cup D$.

Axiom SWC requires that there be no different levels of desirability (undesirability) concerning singletons. In terms of the idea of simple categorization that we want to model, this axiom says that categorization is "simple" in the sense that once an element has been recognized as "desirable" or "undesirable", the agent is unable to discriminate its degree of desirability/undesirability. This kind of simplicity in categorization closely connects with the literature on categorization and stereotypes in social psychology. Furthermore, as already announced in the Introduction, it is particularly suitable under certain interpretations of the sets. For example, in decision problems that involve evaluation of candidates for a group, individual preferences are very often based uniquely on binary criteria such as membership or not of the same party, possession or not of a a certain qualification, etc. As applied to desirable singletons, SWC is very similar to the indifference between no choice situations (INCS) axiom proposed by Pattanaik and Xu (1990) for the evaluation of intrinsic value of freedom of choice that an opportunity set provides. According to INCS any two singletons are indifferent because either provides the same degree of freedom (e.g. no freedom at all).

Axiom SAC formally expresses another aspect of the kind of simplicity that we propose for categorization. In words it states that if a singleton is preferred to the empty set, and another singleton is worse than the empty set, then a two-element set consisting of both elements should be indifferent to the empty set. To put it shortly: The value of any desirable element is always offset by the value of any undesirable element, and vice-versa. The type of ease of categorization that the axiom expresses can be interpreted in different ways: For example it can be understood as if the axiom establishes some kind of equidistance between the category of desirable elements and the category of undesirable elements. It could also be interpreted as assuming that neither category is stronger than the other and that they are symmetric with respect to the reference point of the empty set. Though there are different settings where the axiom makes sense (for example, when a voting process is involved), we are aware that the axiom may be unsuitable in other contexts. This leads us to develop a thorough analysis of the formal implications of relaxing SAC in Sect. 4.3 below.

Axiom IN does not reflect any particular notion of simplicity in categorization, but it does play an important role as a kind of separability condition between the elements of the sets, thus preventing any kind of cross-effects between them. Formally, it requires that adding to (or removing from) two sets $A$ and $B$ respective indifferent sets $C$ and $D$ should not affect the primitive relation between $A$ and $B$. Axiom IN is very close to one of the three axioms that de Finetti (1931) proposes as necessary in the qualitative probability measuring problem. However, similar conditions are very common in ranking sets models under different scenarios. 
It is easy to see that IN implies the following properties:

$$
\begin{aligned}
& \forall a, b \in X,(\{a\} \succ \varnothing \text { and }\{b\} \succ \varnothing) \text { implies }\{a, b\} \succ \varnothing . \\
& \forall a, b \in X,(\varnothing \succ\{a\} \text { and } \varnothing \succ\{b\}) \text { implies } \varnothing \succ\{a, b\} . \\
& \forall a, b \in X,(\{a\} \sim \varnothing \text { and }\{b\} \sim \varnothing) \text { implies }\{a, b\} \sim \varnothing .
\end{aligned}
$$

We check that axioms SWC, SAC, and IN are independent in Sect. 4.1 below. Nevertheless there is a logical relationship between them, which we reveal in Sect. 4.2 below: Under a fairly weak requirement of the domain of the problem (that there exist at least one element in $X$ labeled as good and one other labeled as bad), SAC and IN imply SWC.

\section{Characterization results}

\subsection{The GNB rule}

We are now in a position to characterize GNB rules (cf. Definition 2) as follows. ${ }^{4}$

Theorem 4.1 Let $\succcurlyeq \in \xi$. Then $\succcurlyeq$ satisfies IN, SAC and SWC if and only if $\succcurlyeq$ is a GNB ranking.

In order to prove the theorem we make use of the following lemma, which states some implications of IN and SAC.

Lemma 4.2 Let $\succcurlyeq \in \xi . I f \succcurlyeq$ satisfies IN and SAC then the following two properties hold true.

(a) For all $A \in \chi_{0}: A \succcurlyeq \varnothing$ if and only if $\sum_{a \in A} \mathbf{c}(a) \geqslant 0$.

(b) For all $A \in \chi_{0}: \varnothing \succcurlyeq A$ if and only if $\sum_{a \in A} \mathbf{c}(a) \leqslant 0$.

Proof It suffices to prove (a). We use a recursive argument on the cardinality of $A$. The definition of a characteristic function means that (a) holds for sets with cardinality 1. Assume that (a) is true for sets with cardinality less than $n$, and that $A \in \chi_{0}$ has $n$ elements. We need to check that either both $A \succcurlyeq \varnothing$ and $\sum_{a \in A} \mathbf{c}(a) \geqslant 0$ are true, or both are false. Two cases arise.

Case 1: For all $a \in A,\{a\} \succcurlyeq \varnothing$. A recursive argument based on IN and transitivity of $\succcurlyeq$ yields $A \succcurlyeq \varnothing$. Thus both $A \succcurlyeq \varnothing$ and $\sum_{a \in A} \mathbf{c}(a) \geqslant 0$ are true, and the equivalence (a) is valid.

Case 2: Case 1 does not hold. This means that there is $a \in A$ such that $\varnothing \succ\{a\}$.

If there is no $a^{\prime} \in A$ such that $\left\{a^{\prime}\right\} \succ \varnothing$ then a recursive argument based on IN and transitivity analogous to that used in Case 1 yields $\varnothing \succ A$. Since $\sum_{a \in A} \mathbf{c}(a)<0$ both $A \succcurlyeq \varnothing$ and $\sum_{a \in A} \mathbf{c}(a) \geqslant 0$ are false, and the equivalence $(a)$ is true.

\footnotetext{
${ }^{4}$ The results throughout this subsection are valid if $X$ is either finite or infinite, and $\chi_{0}$ is the set of finite subsets of $X$, including the empty set.
} 
Assume now that there is $a^{\prime} \in A$ such that $\left\{a^{\prime}\right\} \succ \varnothing$. Since $\left\{a, a^{\prime}\right\} \sim \varnothing$ due to SAC, axiom IN yields

$$
A=\left(A-\left\{a, a^{\prime}\right\}\right) \cup\left\{a, a^{\prime}\right\} \sim A-\left\{a, a^{\prime}\right\} .
$$

Moreover, a simple computation shows that

$$
\sum_{b \in A} \mathbf{c}(b)=\left(\sum_{b \in A-\left\{a, a^{\prime}\right\}} \mathbf{c}(b)\right)+\mathbf{c}(a)+\mathbf{c}\left(a^{\prime}\right)=\sum_{b \in A-\left\{a, a^{\prime}\right\}} \mathbf{c}(b)
$$

Summing up: the equivalence $\left(A \succcurlyeq \varnothing \Leftrightarrow \sum_{a \in A} \mathbf{c}(a) \geqslant 0\right)$ is true if and only if the equivalence $\left(A-\left\{a, a^{\prime}\right\} \succcurlyeq \varnothing \Leftrightarrow \sum_{a \in A-\left\{a, a^{\prime}\right\}} \mathbf{c}(a) \geqslant 0\right)$ is true. By the inductive hypothesis, the proof is complete.

Proof of Theorem 4.1 Necessity is immediately apparent. Sufficiency remains to be proved, that is, if $\succcurlyeq$ satisfies IN, SAC and SWC then the equivalence (1) holds true. Let $A, B \in \chi_{0}$. It must be checked that $\sum_{a \in A} \mathbf{c}(a) \geqslant \sum_{b \in B} \mathbf{c}(b)$ holds true.

It can be assumed that $\forall A^{\prime}, B^{\prime} \in \chi_{0}$,

$$
\forall a \in A^{\prime}, \forall b \in B^{\prime}, \quad \neg[\{a\} \sim\{b\}]
$$

The reason is as follows. Due to IN, whenever $a \in A^{\prime}, b \in B^{\prime}$ and $\{a\} \sim\{b\}$ it can be checked that the equivalence $\left(A^{\prime} \succcurlyeq B^{\prime} \Leftrightarrow \sum_{a \in A^{\prime}} \mathbf{c}(a) \geqslant \sum_{b \in B^{\prime}} \mathbf{c}(b)\right)$ is true if and only if the equivalence $\left(A^{\prime}-\{a\} \succcurlyeq B^{\prime}-\{b\} \Leftrightarrow \sum_{a \in A^{\prime}-\{a\}} \mathbf{c}(a) \geqslant \sum_{b \in B^{\prime}-\{b\}} \mathbf{c}(b)\right)$ is true. It is then possible to proceed recursively until (2) is met by a suitable pair of subsets of $A^{\prime}$ and $B^{\prime}$.

Now three cases arise.

Case 1: There is $a \in A$ such that $\{a\} \succ \varnothing$.

If $\{b\} \sim \varnothing$ for all $b \in B$ (which includes the case $B=\varnothing$ ) then the proof is complete by $(a)$ in Lemma 4.2 .

Assume therefore that there is $b \in B$ such that $\neg[\{b\} \sim \varnothing]$. Because (2) and SWC together exclude $\{b\} \succ \varnothing$, the consequence $\varnothing \succ\{b\}$ follows. Again by (2) and SWC, $\left\{a^{\prime}\right\} \succcurlyeq \varnothing$ must hold for each $a^{\prime} \in A$. Now both $A \succcurlyeq \varnothing \succcurlyeq B$ and $\sum_{a \in A} \mathbf{c}(a) \geqslant 0 \geqslant \sum_{b \in B} \mathbf{c}(b)$ follow. Therefore the equivalence (1) holds true.

Case 2: There is $a \in A$ such that $\varnothing \succ\{a\}$.

This case is similar to Case 1 , but now the equivalence (1) holds true because both $A \succcurlyeq B$ and $\sum_{a \in A} \mathbf{c}(a) \geqslant \sum_{b \in B} \mathbf{c}(b)$ are false.

Case 3: If Case 1 and Case 2 are not met, then $a \in A \Rightarrow\{a\} \sim \varnothing$ (this includes the case $A=\varnothing)$.

Now statement (1) boils down to $A \sim \varnothing \succcurlyeq B$ if and only if $0 \geqslant \sum_{b \in B} \mathbf{c}(b)$. This holds by $(b)$ in Lemma 4.2 .

The following examples show that the characterization is tight, that is, that the three axioms in Theorem 4.1 are logically independent. 
1. Let $X=\left\{x, y_{1}, y_{2}\right\}$ and consider the following rule: $\forall A, B \in \chi_{0}, A \succcurlyeq B \Leftrightarrow$ $\sum_{a \in A} u(a) \geqslant \sum_{b \in B} u(b)$, with $u(x)=3, u\left(y_{1}\right)=u\left(y_{2}\right)=1$. The rule satisfies IN, and SAC holds vacuously. However, SWC does not hold true since $\{x\} \succ$ $\left\{y_{1}\right\} \succ \varnothing$.

2. Let $X=\left\{x_{1}, x_{2}, y_{1}, y_{2}\right\}$, and consider a rule as before according to the following values of $u: u\left(x_{1}\right)=u\left(x_{2}\right)=2, u\left(y_{1}\right)=u\left(y_{2}\right)=-1$. Again, the rule satisfies IN and SWC. However, it is plain that SAC is not satisfied.

3. Let $X=\{x, y, z\}$ and $\succcurlyeq$ defined on $\chi_{0}$ be such that $\{x, y, z\} \succ\{x, y\} \sim\{x\} \sim$ $\{y\} \succ \varnothing \sim\{x, z\} \sim\{y, z\} \succ\{z\}$. Then $\succcurlyeq$ satisfies SWC and SAC, but not IN since for example, $\{x\} \succ\{x, z\}$ but not $\{x, y\} \succ\{x, y, z\}$.

In the subsections below we are concerned with particular specifications of the axiomatic structure identified by Theorem 4.1 and the consequences of dropping or relaxing one of the three axioms. To that end we point out that dropping IN leads to rather uninteresting rules: It is easy to check that by only imposing SWC and SAC comparisons between two sets $A, B$ can be established only if each of them is either the empty set, a singleton or contains exactly one "good" and one "bad" element. We therefore skip that analysis. In Sect. 4.2 we explore what happens when SWC is dropped or relaxed, and in Sect. 4.3 we perform a parallel analysis when the axiom dropped is SAC.

\subsection{The restricted-cardinality based rule}

In order to explore the rules that satisfy axioms IN and SAC, we first emphasize the fact that axiom SWC in Theorem 4.1 is superfluous if we assume that the domain is minimally rich, namely, if there exist at least one good and one bad element. We state this formally by means of the following Lemma. Moreover, this result allows to propose an alternative to Theorem 4.1 in the form of a Corollary.

Lemma 4.3 Let $\succcurlyeq \in \xi$ and assume that there exist $x, y \in X$ such that $\{x\} \succ \varnothing \succ\{y\}$. If $\succcurlyeq$ satisfies IN and SAC then it satisfies SWC.

Proof If $(\{a\} \succ \varnothing$ and $\{b\} \succ \varnothing)$ then SAC entails $\{y, a\} \sim \varnothing$ and $\{y, b\} \sim \varnothing$, and now transitivity and IN yield $\{a\} \sim\{b\}$. Likewise, if $(\varnothing \succ\{a\}$ and $\varnothing \succ\{b\})$ then $\{x, a\} \sim \varnothing$ and $\{x, b\} \sim \varnothing$, thus $\{a\} \sim\{b\} .{ }^{5}$

Corollary 4.4 Let $\succcurlyeq \in \xi$ and assume that there exist $x, y \in X$ with $\{x\} \succ \varnothing \succ\{y\}$. Then $\succcurlyeq$ satisfies IN and SAC if and only if $\succcurlyeq$ is a GNB ranking.

To summarize the formal consequences of dropping SWC, that is, the exact implications of SAC and IN, two cases can be distinguished: (i) There exist $x, y \in X$ such that $\{x\} \succ \varnothing \succ\{y\}$, in which case SWC turns out to be a derived axiom and GNB rules can thus be characterized solely by SAC and IN. And (ii) either $\{x\} \succcurlyeq \varnothing \forall x \in X$ or $\varnothing \succcurlyeq\{x\} \forall x \in X$, in which case SAC is a vacuous axiom, and then dropping SWC just leads to the family of binary relations over sets that satisfy the independence axiom.

\footnotetext{
${ }^{5}$ It should be noted that a weaker version of IN, where both $C$ and $D$ are singletons, also allows the Lemma to be proved.
} 
Let us now explore the following class of rules. The ranking $\succcurlyeq$ over the subsets of $X$ is a restricted-cardinality based rule (henceforth RCB) if a subset $X_{S} \subseteq X$ exists such that $A \succcurlyeq B$ if and only if $\left|A \cap X_{S}\right| \geqslant\left|B \cap X_{S}\right|$. Alcantud and Arlegi (2008) discuss this ranking widely and provide several economic contexts where it is suitable (especially for the problem of ranking opportunity sets). It can be easily checked that RCB rules are a particular case of GNB rules with no "bad" elements in the domain. Formally, assume that there is no $x \in X$ such that $\varnothing \succ\{x\}$, and let us define $X_{s}$ by $X_{s}=\{x \in X$ such that $\{x\} \succ \varnothing\}$. It turns out that if $\succcurlyeq$ is a RCB rule, then $A \succcurlyeq B \Leftrightarrow\left|A \cap X_{s}\right| \geqslant\left|B \cap X_{s}\right| \Leftrightarrow \sum_{a \in A} \mathbf{c}(a) \geqslant \sum_{b \in B} \mathbf{c}(b)$. In other words, an $\mathrm{RCB}$ rule is a particular case of a GNB rule where the range of its characteristic function $\mathbf{c}$ is restricted to $\{0,1\}$.

Alcantud and Arlegi (2008) characterize that class of rules axiomatically through the following axioms:

- Non-negativity. For all $A \in \chi_{0}, A \succcurlyeq \varnothing$.

- Symmetry between significant elements: $\{x\} \succ \varnothing$ and $\{y\} \succ \varnothing$ imply $\{x\} \sim\{y\}$, for each $x, y \in X$.

- Simple independence: For every $A, B \in \chi_{0}$ and $x \notin A \cup B, A \succcurlyeq B$ if and only if $A \cup\{x\} \succcurlyeq B \cup\{x\}$.

Theorem 4.5 (Alcantud and Arlegi 2008) Let $\succcurlyeq$ be a complete and transitive binary relation defined on $\chi_{0}$. Then:

$\succcurlyeq$ is RCB if and only if it satisfies non negativity, simple independence and symmetry between significant elements.

At this point a comparison with the axioms that characterize GNB rules is in order. On the one hand, it is clear that symmetry between significant elements and simple independence are weaker versions of SWC and IN respectively. As for non-negativity, this axiom just makes explicit the restriction referred to above that no bad sets (and therefore singletons) are conceivable. As already pointed out, there are various decisional contexts where such an assumption is suitable. As a matter of fact, like IN, Non-negativity is also proposed by de Finetti (1931) as a necessary condition for ranking events in terms of their qualitative probability. In this case, the axiom just expresses that no event can be perceived as less probable than the empty event.

The fact that an RCB rule is a particularization of a GNB rule means that it must be possible to subsume the axioms that characterize the RCB rule as particularizations of those that characterize the GNB rule under the assumption that there is no $x \in X$ such that $\varnothing \succ\{x\}$. Indeed, under such an assumption, it is easy to see that SWC becomes "symmetry between significant elements", SAC holds vacuously, and "simple independence" is a weaker version of IN.

\subsection{The weighted GNB rule}

Under the assumption that there exist $x, y \in X$ with $\{x\} \succ \varnothing>\{y\}$, the precise implications of SAC plus IN are established in Corollary 4.4: they hold exactly in the presence of GNB behavior. In this Section we analyze the precise implications of SWC together with IN. We check that they hold exactly in the presence of a weighted 
GNB behavior, i.e. behavior that attaches possibly different weights to good and bad elements. To that end we introduce further notation and prove Theorem 4.6 below.

For all $A \subseteq X, \mathbf{g}(A)=\{a \in A:\{a\} \succ \varnothing\}$, and $\mathbf{b}(A)=\{a \in A: \varnothing \succ\{a\}\}$. The sets $\mathbf{g}(A)$ and $\mathbf{b}(A)$ are interpreted as the subsets of good and bad elements of $A$ respectively. Observe that $\sum_{a \in A} \mathbf{c}(a)=|\mathbf{g}(A)|-|\mathbf{b}(A)|$ for all $A \in \chi_{0}$.

When $A, B \in \chi_{0}$ are such that $|\mathbf{g}(A)| \neq|\mathbf{g}(B)|, \sigma_{A B}$ denotes the value $\frac{|\mathbf{b}(A)|-|\mathbf{b}(B)|}{|\mathbf{g}(A)|-|\mathbf{g}(B)|}$. Of course, $\sigma_{A B}=\sigma_{B A}$ throughout.

Finally, we let $S(A)=\left\{B \in \chi_{0}:|\mathbf{g}(A)|>|\mathbf{g}(B)|\right.$ and $\left.|\mathbf{b}(A)|>|\mathbf{b}(B)|\right\}$.

Definition 3 We call the ranking of sets $\succcurlyeq$ WGNB (or weighted GNB) if there exist $\alpha, \beta \in \mathbb{R}_{++}$such that $A \succcurlyeq B \Leftrightarrow \alpha|\mathbf{g}(A)|-\beta|\mathbf{b}(A)| \geqslant \alpha|\mathbf{g}(B)|-\beta|\mathbf{b}(B)|$ for all $A, B \in \chi_{0}$.

Clearly, GNB rules are WGNB rules where $\alpha=\beta$. Our next Theorem characterizes the more general family of WGNB rules axiomatically:

Theorem 4.6 Let $\succcurlyeq$ be an ordering on $\chi_{0}$. Then $\succcurlyeq$ satisfies IN and SWC if and only if $\succcurlyeq$ is $W G N B$.

The fact that WGNB rules agree with IN and SWC can be easily checked. We proceed to prove the converse implication, for which several lemmata are proved under the common assumptions IN and SWC.

Lemma 4.7 For all $A \in \chi_{0}, A \sim A \backslash\{x \in A:\{x\} \sim \varnothing\}$.

Proof Denote $\{x \in A:\{x\} \sim \varnothing\}$ by $\left\{x_{1}, \ldots, x_{n}\right\}$. By IN $x_{n} \sim \varnothing$ implies $\left\{x_{1}, \ldots, x_{n}\right\} \sim\left\{x_{1}, \ldots, x_{n-1}\right\}$. Repeating the argument $n$-times, and by transitivity, $\left\{x_{1}, \ldots, x_{n}\right\} \sim \varnothing$ is obtained. Now IN permits the conclusion that $A \sim$ $A \backslash\left\{x_{1}, \ldots, x_{n}\right\}$.

For the sake of proving Theorem 4.6 below, Lemma 4.7 allows $\{x \in A:\{x\} \sim$ $\varnothing\}=\varnothing$ to be assumed for all $A \in \chi_{0}$ henceforth in this Section.

Lemma 4.8 For all $A, B \in \chi_{0},|\mathbf{g}(A)|=|\mathbf{g}(B)|$ and $|\mathbf{b}(A)|=|\mathbf{b}(B)|$ implies $A \sim B$.

Proof Let $A=\left\{g_{1}(A), \ldots, g_{m}(A), b_{1}(A), \ldots, b_{n}(A)\right\}, B=\left\{g_{1}(B), \ldots, g_{m}(B)\right.$, $\left.b_{1}(B), \ldots, b_{n}(B)\right\}$, where $\mathbf{g}(A)=\left\{g_{1}(A), \ldots, g_{m}(A)\right\}$ and $\mathbf{b}(A)=\left\{b_{1}(A), \ldots\right.$, $\left.b_{n}(A)\right\}, \mathbf{g}(B)=\left\{g_{1}(B), \ldots, g_{m}(B)\right\}$ and $\mathbf{b}(B)=\left\{b_{1}(B), \ldots, b_{n}(B)\right\}$. By SWC $b_{i}(A) \sim b_{i}(B)$ for all $i \leqslant n$, and IN can be applied $n$ times to obtain that $\mathbf{b}(A) \sim \mathbf{b}(B)$. Also by SWC $g_{i}(A) \sim g_{i}(B)$ for all $i \leqslant m$. Observe that $(\mathbf{g}(A) \cup \mathbf{g}(B)) \cap(\mathbf{b}(A) \cup$ $\mathbf{b}(B))=\varnothing$. Thus, IN can be applied $m$ times analogously to obtain $\mathbf{g}(A) \cup \mathbf{b}(A) \sim$ $\mathbf{g}(B) \cup \mathbf{b}(B)$, that is, $A \sim B$.

Lemma 4.9 For all $A, B \in \chi_{0}$, if $|\mathbf{g}(A)|=|\mathbf{g}(B)|$ and $|\mathbf{b}(A)|>|\mathbf{b}(B)|$ then $B \succ A$.

Proof Start by assuming that $|\mathbf{b}(A)|=|\mathbf{b}(B)|+1$. Let $A=\left\{g_{1}(A), \ldots, g_{m}(A)\right.$, $\left.b_{1}(A), \ldots, b_{n+1}(A)\right\}$. With no loss of generality $b_{n+1}(A) \notin \mathbf{b}(B)$. Lemma 4.8 yields $B \sim A \backslash\left\{b_{n+1}(A)\right\}$, and a direct argument based on IN proves $B \succ A$. 
In the general case, a recursive argument based on the instance above yields the thesis.

Lemma 4.10 For all $A, B \in \chi_{0}$, if $|\mathbf{g}(A)|>|\mathbf{g}(B)|$ and $|\mathbf{b}(A)|=|\mathbf{b}(B)|$ then $A \succ B$.

Proof Analogous to Lemma 4.9.

From Lemmata 4.8-4.10, for all $A, B \in \chi_{0}$ the following holds:

$$
\begin{aligned}
& \text { if }|\mathbf{g}(A)| \geqslant|\mathbf{g}(B)| \text { and }|\mathbf{b}(A)| \leqslant|\mathbf{b}(B)| \text { then every } \alpha, \beta \in \mathbb{R}_{++} \text {verify } \\
& A \succcurlyeq B \Leftrightarrow \alpha|\mathbf{g}(A)|-\beta|\mathbf{b}(A)| \geqslant \alpha|\mathbf{g}(B)|-\beta|\mathbf{b}(B)|
\end{aligned}
$$

Lemma 4.11 Forall $A, B, C, D \in \chi_{0}$ such that $B \in S(A), D \in S(C)$, and $\frac{|\mathbf{b}(A)|-|\mathbf{b}(B)|}{|\mathbf{g}(A)|-|\mathbf{g}(B)|}=$ $\frac{|\mathbf{b}(C)|-|\mathbf{b}(D)|}{|\mathbf{g}(C)|-|\mathbf{g}(D)|}: A \succcurlyeq B \Leftrightarrow C \succcurlyeq D$.

Proof For any pair of sets $V, W \in \chi_{0}$ such that $W \in S(V)$, if $p, q \in \mathbb{N}$ are such that $\frac{p}{q}$ is an irreducible fraction and $\frac{p}{q}=\sigma_{V W}$ then there exists $n \in\{1,2,3, \ldots\}$ with $|\mathbf{b}(V)|-|\mathbf{b}(W)|=p n$ and $|\mathbf{g}(V)|-|\mathbf{g}(W)|=q n$.

It is clearly possible to construct $E \in \chi_{0}$ with the properties $|\mathbf{b}(E)|=p,|\mathbf{b}(E)|=$ $q, E \cap W=\varnothing, E \subseteq V$. By Lemma 4.8, any other $E^{\prime}$ with $\left|\mathbf{b}\left(E^{\prime}\right)\right|=p$ and $\left|\mathbf{b}\left(E^{\prime}\right)\right|=q$ satisfies $E \sim E^{\prime}$.

The argument above assures that our Lemma reduces to the following property: $E \succ \varnothing$ implies $V \succ W ; \varnothing \succ E$ implies $W \succ V$; and $\varnothing \sim E$ implies $W \sim V$. We prove this equivalent property by a recursive construction.

Let $W_{1}=W \cup E$. When $n=1$, this set coincides with $V$ thus the thesis follows directly from IN. In all cases, an appeal to this axiom yields that $E \succ \varnothing$ if and only if $W_{1} \succ W$, and also $\varnothing \succ E$ if and only if $W \succ W_{1}$.

$E_{1} \in \chi_{0}$ can also be constructed with the properties $\left|\mathbf{b}\left(E_{1}\right)\right|=p,\left|\mathbf{b}\left(E_{1}\right)\right|=q$, $E_{1} \cap W_{1}=\varnothing$, and $E_{1} \subseteq V$.

Let $W_{2}=W_{1} \cup E$. Reasoning as above we conclude as follows:

$$
\begin{aligned}
& W_{2} \succ W_{1} \Leftrightarrow E_{1} \succ \varnothing \Leftrightarrow E \succ \varnothing \Leftrightarrow W_{1} \succ W, \text { thus } E \succ \varnothing \Rightarrow W_{2} \succ W . \\
& W_{1} \succ W_{2} \Leftrightarrow \varnothing \succ E_{1} \Leftrightarrow \varnothing \succ E \Leftrightarrow W \succ W_{1}, \text { thus } \varnothing \succ E \Rightarrow W \succ W_{2} . \\
& W_{2} \sim W_{1} \Leftrightarrow E_{1} \sim \varnothing \Leftrightarrow E \sim \varnothing \Leftrightarrow W_{1} \sim W, \text { thus } E \sim \varnothing \Rightarrow W_{2} \sim W .
\end{aligned}
$$

In case $n=2$ the argument ends because $W_{2}=V$.

By repeating this construction we reach $W_{n}=V$, which satisfies the property $E \succ \varnothing \Rightarrow W_{n} \succ W, \varnothing \succ E \Rightarrow W \succ W_{n}$, and $E \sim \varnothing \Rightarrow W_{n} \sim W$ as was claimed.

Proof of Theorem 4.6 We first argue that the property that we must check can be simplified. As argued in (3), we can narrow our interest to the pairs of sets $A, B \in \chi_{0}$ such that either $A \in S(B)$ or $B \in S(A)$ since no restriction is imposed by the cases where $|\mathbf{g}(A)| \geqslant|\mathbf{g}(B)|$ and $|\mathbf{b}(A)| \leqslant|\mathbf{b}(B)|$.

Observe further that if $A \succ B$ for all $A, B \in \chi_{0}$ such that $B \in S(A)$, then the theorem is proved by taking positive $\alpha, \beta$ such that $\frac{\alpha}{\beta}>|X|$. Analogously, if $B \succ A$ 
for all $A, B \in \chi_{0}$ such that $B \in S(A)$ then the theorem is proved by taking positive $\alpha, \beta$ such that $\frac{\alpha}{\beta}<\frac{1}{|X|} \cdot 6$

In view of these facts, there is no loss of generality if it is assumed that there exist $A, B, C, D \in \chi_{0}$ such that $B \in S(A), D \in S(C), A \succ B$ and $D \succ C$.

Now, in order to proceed with the proof of the Theorem two cases appear:

Case 1: There do not exist $A, B \in \chi_{0}$ such that $|\mathbf{b}(A)| \neq|\mathbf{b}(B)|,|\mathbf{g}(A)| \neq|\mathbf{g}(B)|$ and $A \sim B$.

Define the following values:

$\sigma_{1}=\max \left\{\frac{|\mathbf{b}(A)|-|\mathbf{b}(B)|}{|\mathbf{g}(A)|-|\mathbf{g}(B)|}: A \succ B, B \in S(A)\right\}=\max \left\{\sigma_{A B}: A \succ B, B \in S(A)\right\}$

and

$\sigma_{2}=\min \left\{\frac{|\mathbf{b}(A)|-|\mathbf{b}(B)|}{|\mathbf{g}(A)|-|\mathbf{g}(B)|}: B \succ A, B \in S(A)\right\}=\min \left\{\sigma_{A B}: B \succ A, B \in S(A)\right\}$.

We prove that $\sigma_{1}<\sigma_{2}$. Observe that $\sigma_{1}=\sigma_{2}$ is against Lemma 4.11, therefore it suffices to prove that $\sigma_{1}>\sigma_{2}$ leads to a contradiction.

We take sets $A, B$ with $B \in S(A), A \succ B$ at which the maximum $\sigma_{1}$ is attained, that is, $A, B$ verify $\frac{|\mathbf{b}(A)|-|\mathbf{b}(B)|}{|\mathbf{g}(A)|-|\mathbf{g}(B)|} \geqslant \frac{|\mathbf{b}(F)|-|\mathbf{b}(G)|}{|\mathbf{g}(F)|-|\mathbf{g}(G)|}$ for all $F, G \in \chi_{0}$ such that $F \succ G$ and $G \in S(F)$. Analogously, we also take sets $C, D$ with $D \in S(C), D \succ C$, at which the maximum $\sigma_{2}$ is attained.

We write $\sigma_{1}=\frac{p}{q}, \sigma_{2}=\frac{r}{t}$, both irreducible fractions, and $p, q, r, t>0$. It should be noted that $A^{\prime}$ and $B$ can be always chosen so that $\left|\mathbf{b}\left(A^{\prime}\right)\right|-|\mathbf{b}(B)|=p$ and $\left|\mathbf{g}\left(A^{\prime}\right)\right|-|\mathbf{g}(B)|=q$, and similarly $C^{\prime}$ and $D$ can be chosen so that $\left|\mathbf{b}\left(C^{\prime}\right)\right|-|\mathbf{b}(D)|=r$ and $\left|\mathbf{g}\left(C^{\prime}\right)\right|-|\mathbf{g}(D)|=t$. The first claim is justified by removing the appropriate number of elements from $A$ in order to obtain a set $A^{\prime}$ that satisfies $\sigma_{A^{\prime} B}=\sigma_{A B}$ and $B \in$ $S\left(A^{\prime}\right)$, and the second claim is justified similarly. Then, in accordance to Lemma 4.11 $A \succ B$ if and only if $A^{\prime} \succ B$ and $C \succ D$ if and only if $C^{\prime} \succ D$. Therefore, it is assumed throughout that $|\mathbf{b}(A)|-|\mathbf{b}(B)|=p,|\mathbf{g}(A)|-|\mathbf{g}(B)|=q,|\mathbf{b}(C)|-|\mathbf{b}(D)|=r$ and $|\mathbf{g}(C)|-|\mathbf{g}(D)|=t$.

We distinguish the following exhaustive list of subcases:

Case 1.1: $p \geqslant r$ and $q \leqslant t$ with at least one strict inequality.

In case $p=r$ we let $B^{\prime}=B$, otherwise there is $B_{A} \subseteq \mathbf{b}(A)$ with $\left|B_{A}\right|=p-r$ and $B_{A} \cap \mathbf{b}(B)=\varnothing$ and we let $B^{\prime}=B \cup B_{A}$. In this latter instance Lemma 4.9 yields $B \succ B^{\prime}$. In all cases, given that $A \succ B, A \succ B^{\prime}$ results.

In case $q=t$ we let $D^{\prime}=D$, otherwise there is $G_{C} \subseteq \mathbf{g}(C)$ with $\left|G_{C}\right|=t-q$ and $G_{C} \cap \mathbf{g}(D)=\varnothing$ and we let $D^{\prime}=D \cup G_{C}$. In this latter instance Lemma 4.10 yields $D^{\prime} \succ D$. In all cases, given that $D \succ C, D^{\prime} \succ C$ results.

\footnotetext{
6 Note that in these cases we have two lexicographic rules. The first compares any pair of sets by maximizing the number of good elements, and minimizes the number of bad elements only in case of a tie. The second one is the dual lexicographic rule. In other words, such lexicographic rules are particular cases of WGNB rules for the indicated values of $\alpha$ and $\beta$.
} 
By construction $\frac{|\mathbf{b}(A)|-\left|\mathbf{b}\left(B^{\prime}\right)\right|}{|\mathbf{g}(A)|-\left|\mathbf{g}\left(B^{\prime}\right)\right|}=\frac{|\mathbf{b}(C)|-\left|\mathbf{b}\left(D^{\prime}\right)\right|}{|\mathbf{g}(C)|-\left|\mathbf{g}\left(D^{\prime}\right)\right|}=\frac{r}{q}$. Because $B^{\prime} \in S(A)$ and $D^{\prime} \in$ $S(C)$, Lemma 4.11 assures $A \succ B^{\prime} \Leftrightarrow C \succ D^{\prime}$, which leads to a contradiction.

Case 1.2: $p>r$ and $q>t$.

In this case some simple computations prove that $\frac{p-r}{q-t}>\frac{p}{q}$. Now there is $B_{A} \subseteq$ $\mathbf{b}(A)$ with $\left|B_{A}\right|=p-r$ and $B_{A} \cap \mathbf{b}(B)=\varnothing$, and also there is $G_{A} \subseteq \mathbf{g}(A)$ with $\left|G_{A}\right|=q-t$ and $G_{A} \cap \mathbf{g}(B)=\varnothing$. Let $B^{\prime}=B \cup B_{A} \cup G_{A}$.

By construction $\frac{\left|\mathbf{b}\left(B^{\prime}\right)\right|-|\mathbf{b}(B)|}{\left|\mathbf{g}\left(B^{\prime}\right)\right|-|\mathbf{g}(B)|}=\frac{p-r}{q-t}>\frac{p}{q}$. By the definition of $\sigma_{1}$ as a maximum, because there are no indifferences among sets with different number of both good and bad elements and $B \in S\left(B^{\prime}\right)$, necessarily $B \succ B^{\prime}$.

Again, by construction $\frac{|\mathbf{b}(A)|-\left|\mathbf{b}\left(B^{\prime}\right)\right|}{|\mathbf{g}(A)|-\left|\mathbf{g}\left(B^{\prime}\right)\right|}=\frac{r}{t}=\frac{|\mathbf{b}(C)|-|\mathbf{b}(D)|}{|\mathbf{g}(C)|-|\mathbf{g}(D)|}$. By Lemma 4.11 we deduce $B^{\prime} \succ A$, and because $A \succcurlyeq B$, transitivity yields the contradiction $B^{\prime} \succ B$.

Case 1.3: $p<r$ and $q<t$.

In this case $\frac{r-p}{t-q}<\frac{r}{t}$. Now there is $B_{C} \subseteq \mathbf{b}(C)$ with $\left|B_{C}\right|=p$ and $B_{C} \cap \mathbf{b}(D)=\varnothing$, and also there is $G_{C} \subseteq \mathbf{g}(C)$ with $\left|G_{C}\right|=q$ and $G_{C} \cap \mathbf{g}(D)=\varnothing$. Let $C^{\prime}=$ $D \cup B_{C} \cup G_{C}$.

By construction $\frac{|\mathbf{b}(C)|-\left|\mathbf{b}\left(C^{\prime}\right)\right|}{|\mathbf{g}(C)|-\left|\mathbf{g}\left(C^{\prime}\right)\right|}=\frac{r-p}{t-q}$ and $C^{\prime} \in S(C)$. Since $\frac{r-p}{t-q}<\frac{r}{t}$ and there are no indifferences between sets with different numbers of both good and bad elements, and by the definition of $\sigma_{2}$ as a minimum, we have that necessarily $C \succ C^{\prime}$, and by transitivity $D \succ C^{\prime}$.

Also, by definition $\frac{\left|\mathbf{b}\left(C^{\prime}\right)\right|-|\mathbf{b}(D)|}{\left|\mathbf{g}\left(C^{\prime}\right)\right|-|\mathbf{g}(D)|}=\frac{p}{q}=\frac{|\mathbf{b}(A)|-|\mathbf{b}(B)|}{|\mathbf{g}(A)|-|\mathbf{g}(B)|}$. By Lemma 4.11 that implies $C^{\prime} \succ D$, leading to a contradiction.

Since all three of the possible cases above lead to a contradiction we conclude that $\sigma_{1}<\sigma_{2}$. This implies that there do not exist $E, F \in \chi_{0}$ with $F \in S(E)$ such that $\sigma_{1}<\frac{|\mathbf{b}(E)|-|\mathbf{b}(F)|}{|\mathbf{g}(E)|-|\mathbf{g}(F)|}<\sigma_{2}$ and $E \succ F$ or $F \succ E$. Since there is no indifference between pairs of sets with different numbers of both good and bad elements, for all $E, F \in \chi_{0}$ with $F \in S(E)$ either $\frac{|\mathbf{b}(E)|-|\mathbf{b}(F)|}{|\mathbf{g}(E)|-|\mathbf{g}(F)|} \leqslant \sigma_{1}$ or $\frac{|\mathbf{b}(E)|-|\mathbf{b}(F)|}{|\mathbf{g}(E)|-|\mathbf{g}(F)|} \geqslant \sigma_{2}$ holds true.

Take any pair of numbers $\alpha, \beta \in \mathbb{R}_{++}$such that $\sigma_{1}<\frac{\alpha}{\beta}<\sigma_{2}$. For each $E, F$ with $F \in S(E), \frac{|\mathbf{b}(E)|-|\mathbf{b}(F)|}{|\mathbf{g}(E)|-|\mathbf{g}(F)|}<\frac{\alpha}{\beta}$ entails $E \succ F$, and $\frac{|\mathbf{b}(E)|-|\mathbf{b}(F)|}{|\mathbf{g}(E)|-|\mathbf{g}(F)|}>\frac{\alpha}{\beta}$ entails $F \succ E$, which completes the proof under Case 1 .

Case 2: There exist $A_{0}, B_{0} \in \chi_{0}$, such that $B_{0} \in S\left(A_{0}\right),\left|\mathbf{b}\left(A_{0}\right)\right| \neq\left|\mathbf{b}\left(B_{0}\right)\right|,\left|\mathbf{g}\left(A_{0}\right)\right| \neq$ $\left|\mathbf{g}\left(B_{0}\right)\right|$ and $A_{0} \sim B_{0}$.

Take $\alpha=\left|\mathbf{b}\left(A_{0}\right)\right|-\left|\mathbf{b}\left(B_{0}\right)\right|>0$ and $\beta=\left|\mathbf{g}\left(A_{0}\right)\right|-\left|\mathbf{g}\left(B_{0}\right)\right|>0$. Due to Lemma 4.11, when $C, D \in \chi_{0}$ are such that $D \in S(C)$ and $\frac{|\mathbf{b}(C)|-|\mathbf{b}(D)|}{|\mathbf{g}(C)|-|\mathbf{g}(D)|}=\frac{\alpha}{\beta}$ then $C \sim D$ holds. Thus what remains to prove is the following:

1. $\forall C, D \in \chi_{0}$ such that $D \in S(C)$ and $\frac{|\mathbf{b}(C)|-|\mathbf{b}(D)|}{|\mathbf{g}(C)|-|\mathbf{g}(D)|}>\frac{\alpha}{\beta}, D \succ C$ holds.

2. $\forall C, D \in \chi_{0}$ such that $D \in S(C)$ and $\frac{|\mathbf{b}(C)|-|\mathbf{b}(D)|}{|\mathbf{g}(C)|-|\mathbf{g}(D)|}<\frac{\alpha}{\beta}, C \succ D$ holds.

Define the following values:

$$
\begin{aligned}
\sigma_{1} & =\max \left\{\frac{|\mathbf{b}(A)|-|\mathbf{b}(B)|}{|\mathbf{g}(A)|-|\mathbf{g}(B)|}: A \succcurlyeq B, B \in S(A)\right\} \\
& =\max \left\{\sigma_{A B}: A \succcurlyeq B, B \in S(A)\right\}
\end{aligned}
$$


and

$$
\begin{aligned}
\sigma_{2} & =\min \left\{\frac{|\mathbf{b}(A)|-|\mathbf{b}(B)|}{|\mathbf{g}(A)|-|\mathbf{g}(B)|}: B \succcurlyeq A, B \in S(A)\right\} \\
& =\min \left\{\sigma_{A B}: B \succcurlyeq A, B \in S(A)\right\} .
\end{aligned}
$$

We proceed to prove that $\sigma_{1}=\sigma_{2}=\frac{\alpha}{\beta}$ according to the following steps. Since $B_{0} \sim A_{0}, \sigma_{1}<\sigma_{2}$ is false: $\sigma_{A_{0} B_{0}}=\frac{\alpha}{\beta} \leqslant \sigma_{1}$ because $A_{0} \succcurlyeq B_{0}$, and $\sigma_{A_{0} B_{0}} \geqslant \sigma_{2}$ because $B_{0} \succcurlyeq A_{0}$. We proceed to establish that $\sigma_{1}>\sigma_{2}$ leads to a contradiction through arguments which follow the line of the proof of Case 1. Consequently, $\frac{\alpha}{\beta} \leqslant$ $\sigma_{1}=\sigma_{2} \leqslant \frac{\alpha}{\beta}$ and thus $\sigma_{1}=\sigma_{2}=\frac{\alpha}{\beta}$ as claimed.

Let $\sigma_{1}=\frac{p}{q}$ and $\sigma_{2}=\frac{r}{t}$, both fractions being irreducible, and $p, q, r, t>0$.

We take sets $A, B$ with $B \in S(A), A \succcurlyeq B$ for which $\sigma_{1}$ is attained. We also take sets $C, D$ with $D \in S(C), D \succcurlyeq C$, for which $\sigma_{2}$ is attained. In accordance to Lemma 4.11, there is no loss of generality if we assume $|\mathbf{b}(A)|-|\mathbf{b}(B)|=p$ and $|\mathbf{g}(A)|-|\mathbf{g}(B)|=q$, and also $|\mathbf{b}(C)|-|\mathbf{b}(D)|=r$ and $|\mathbf{g}(C)|-|\mathbf{g}(D)|=t$.

Again we split our argument into the following subcases:

Case 2.1: $p \geqslant r$ and $q \leqslant t$ with at least one strict inequality.

In case $p=r$ we let $B^{\prime}=B$, otherwise there is $B_{A} \subseteq \mathbf{b}(A)$ with $\left|B_{A}\right|=p-r$ and $B_{A} \cap \mathbf{b}(B)=\varnothing$ and we let $B^{\prime}=B \cup B_{A}$. In this latter instance Lemma 4.9 yields $B \succ B^{\prime}$. In conclusion, $A \succ B^{\prime}$ if $p>r$ and $A \succcurlyeq B^{\prime}$ if $p=r$.

In case $q=t$ we let $D^{\prime}=D$, otherwise there is $G_{C} \subseteq \mathbf{g}(C)$ with $\left|G_{C}\right|=t-q$ and $G_{C} \cap \mathbf{g}(D)=\varnothing$ and we let $D^{\prime}=D \cup G_{C}$. In this latter instance Lemma 4.10 yields $D^{\prime} \succ D$. In conclusion, $D^{\prime} \succ C$ if $q<t$ and $D^{\prime} \succcurlyeq C$ if $q=t$.

By construction $\frac{|\mathbf{b}(A)|-\left|\mathbf{b}\left(B^{\prime}\right)\right|}{|\mathbf{g}(A)|-\left|\mathbf{g}\left(B^{\prime}\right)\right|}=\frac{|\mathbf{b}(C)|-\left|\mathbf{b}\left(D^{\prime}\right)\right|}{|\mathbf{g}(C)|-\left|\mathbf{g}\left(D^{\prime}\right)\right|}=\frac{r}{q}$. Therefore, either $p>r$ (thus $A \succ B^{\prime}$ and Lemma 4.11 yields the contradiction $C \succ D^{\prime}$ ) or $q<t$ (thus $D^{\prime} \succ C$ and Lemma 4.11 yields the contradiction $B^{\prime} \succ A$ ).

Case 2.2: $p>r$ and $q>t$. As in Case 1.2, $\frac{p-r}{q-t}>\frac{p}{q}$.

Now there is $B_{A} \subseteq \mathbf{b}(A)$ with $\left|B_{A}\right|=p-r$ and $B_{A} \cap \mathbf{b}(B)=\varnothing$, and also there is $G_{A} \subseteq \mathbf{g}(A)$ with $\left|G_{A}\right|=q-t$ and $G_{A} \cap \mathbf{g}(B)=\varnothing$. Let $B^{\prime}=B \cup B_{A} \cup G_{A}$.

By construction $\frac{\left|\mathbf{b}\left(B^{\prime}\right)\right|-|\mathbf{b}(B)|}{\left|\mathbf{g}\left(B^{\prime}\right)\right|-|\mathbf{g}(B)|}=\frac{p-r}{q-t}>\frac{p}{q}$. The definition of $\sigma_{1}$ implies that one must have $B \succ B^{\prime}$.

Because $\frac{|\mathbf{b}(A)|-\left|\mathbf{b}\left(B^{\prime}\right)\right|}{|\mathbf{g}(A)|-\left|\mathbf{g}\left(B^{\prime}\right)\right|}=\frac{p}{q}=\frac{|\mathbf{b}(C)|-|\mathbf{b}(D)|}{|\mathbf{g}(C)|-|\mathbf{g}(D)|}$, Lemma 4.11 implies $B^{\prime} \succcurlyeq A$, thus, by transitivity, $B \succ A$, contradicting $A \succcurlyeq B$.

Case 2.3: $p<r$ and $q<t$.

In this case $\frac{r-p}{t-q}<\frac{r}{t}$. Now there is $B_{C} \subseteq \mathbf{b}(C)$ with $\left|B_{C}\right|=p$ and $B_{C} \cap \mathbf{b}(D)=\varnothing$, and there is also $G_{C} \subseteq \mathbf{g}(C)$ with $\left|G_{C}\right|=q$ and $G_{C} \cap \mathbf{g}(D)=\varnothing$. Let $C^{\prime}=$ $D \cup B_{C} \cup G_{C}$.

By construction $\frac{|\mathbf{b}(C)|-\left|\mathbf{b}\left(C^{\prime}\right)\right|}{|\mathbf{g}(C)|-\left|\mathbf{g}\left(C^{\prime}\right)\right|}=\frac{r-p}{t-q}$ and $C^{\prime} \in S(C)$. Since $\frac{r-p}{t-q}<\frac{r}{t}$, the definition of $\sigma_{2}$ yields $C \succ C^{\prime}$. Now transitivity implies $D \succ C^{\prime}$.

Because $\frac{\left|\mathbf{b}\left(C^{\prime}\right)\right|-|\mathbf{b}(D)|}{\left|\mathbf{g}\left(C^{\prime}\right)\right|-|\mathbf{g}(D)|}=\frac{p}{q}=\frac{|\mathbf{b}(A)|-|\mathbf{b}(B)|}{|\mathbf{g}(A)|-|\mathbf{g}(B)|}$, Lemma 4.11 implies the contradiction $C^{\prime} \succcurlyeq D$. 
Since $\sigma_{1}=\sigma_{2}$, if $C, D$ are such that $\frac{|\mathbf{b}(C)|-|\mathbf{b}(D)|}{|\mathbf{g}(C)|-|\mathbf{g}(D)|}>\frac{\alpha}{\beta}$ the definition of $\sigma_{1}$ conveys $D \succ C$, and if they are such that $\frac{|\mathbf{b}(C)|-|\mathbf{b}(D)|}{|\mathbf{g}(C)|-|\mathbf{g}(D)|}<\frac{\alpha}{\beta}$ then $C \succ D$. This completes the proof of the theorem.

\section{Final comments and links to existing literature}

We have explored the consequences of imposing different combinations of three meaningful axioms on a ranking of sets of objects, namely independence (IN), symmetry whithin categories (SWC), and symmetry across categories (SAC). We have checked that these axioms are independent, and that the only rule that satisfies them is a GNB rule. In fact, Axiom SWC is redundant when the rule satisfies an easily checkable characteristic: the existence of elements that are ranked above and below the empty set. Dropping SAC produces a natural generalization of the additive GNB rules where the elements that are not "neutral" are weighted by two positive amounts, one associated with being "good" and the other with being "bad".

Alcantud and Arlegi (2008) also seek to explore the formal consequences of the categorization phenomenon for the matter of ranking sets. Roughly speaking, in that article the case under inspection is that of an agent that makes a categorization in a countable number of positive and increasingly better classes, and then evaluates sets additively according to the corresponding values of their elements. Special attention is paid to the RCB rules, i.e. those that only distinguish between desirable and nondesirable objects without further grading. Here we characterize the family of rules that classify the elements into three classes/categories (desirable, neutral, and rejectable) and may allocate a different weight to each category. The particular case where the weights coincide is characterized by the corresponding axioms. We emphasize that the proofs provided for this case (i.e. Sect. 4.1) are valid if $X$ is infinite, and $\chi_{0}$ is the set of finite subsets of $X$ including the empty set. The same is true for Sect. 4.2.

Our model bears some comparison with Fishburn (1992) model of ranking sets on the basis of signed orders. ${ }^{7}$ Fishburn (1992) proposes the notion of self-reflecting signed orders as an aid in estimating preferences between subsets of items on the basis of limited information. The inputs of a signed order are binary comparisons that blend the desirability of excluding items from a subset and including items in it. In particular, if $X^{*}$ is a disjoint copy of $X$, a self-reflecting signed order $\succcurlyeq_{1}$ on $\left(X \cup X^{*}\right)$ is a weak order that compares relative likes and dislikes for items in $X$. For example, $\succcurlyeq_{1}$ allows comparisons such as the following to be made, "including $x$ in a subset is preferred to excluding $y$ from it" $\left(x \succcurlyeq_{1} y^{*}\right)$, "excluding $z$ from a subset is preferred to including $w$ in it" $\left(z^{*} \succcurlyeq_{1} w\right)$, or "including $a$ in a subset is preferred to including $b$ in it" $\left(a \succcurlyeq_{1} b\right)$.

Some natural properties of $\succcurlyeq_{1}$ enable helpful pieces of information to be induced for the purpose of ranking subsets. For example, a partition of the elements of $X$ between positive, indifferent, and negative elements can be obtained according to $x \succ_{1} x^{*}, x \sim_{1} x^{*}$, and $x^{*} \succ_{1} x$ respectively. The model also introduces the idea of

\footnotetext{
7 We thank an anonymous referee for pointing out this reference to us.
} 
a function $f$ that preserves $\succcurlyeq_{1}$, which takes positive (negative/ null) values for good (bad/neutral) items, such that for each $x, y \in X, f(x) \geqslant f(y) \Leftrightarrow x \succcurlyeq_{1} y, f(x) \geqslant$ $-f(y) \Leftrightarrow x \succcurlyeq_{1} y^{*}$ and $-f(x) \geqslant f(y) \Leftrightarrow x^{*} \succcurlyeq_{1} y$. At this point Fishburn (1992) concentrates on additive rankings where $A \succcurlyeq B \Leftrightarrow f(A) \geqslant f(B)$, under the convention $f(S)=\sum_{s \in S} f(S)$ for each subset $S$.

With those elements at hand, the first main result in Fishburn (1992, Theorem 1) enables some subsets to be identified as good, bad, and indifferent with a clear sense of $A \succ \varnothing, \varnothing \succ A, A \sim \varnothing$ on the basis of certain "offsetting" conditions. For example, a set is good if and only if it contains only positive elements or if it contains both positive and negative elements and it is possible to associate each negative element $x$ with a different positive element $y$ in such a way that: (1) either $y \succcurlyeq_{1} x^{*}$ with strict preference for at least one pair, or (2) $y \sim_{1} x^{*}$ for every pair and the number of positive elements in the set is greater than the number of negative elements. Conditions for sets to be bad or indifferent are analogous. ${ }^{8}$

According to the second main result in Fishburn (1992, Theorem 2), some additional comparisons between non empty sets can be established by imposing a new condition, which also involves compensations of values within each set and between the two sets under comparison. ${ }^{9}$

Given that both GNB and WGNB rules are additive, one might think that it is possible to induce an underlying self reflecting signed order in both cases for which Fishburn (1992) results are fulfilled. This is true for GNB rules but not for the general family of WGNB rules. In particular, $f$ can be specified by $f(x)>0$ when $\{x\} \succ \varnothing, f(x)=0$ when $\{x\} \sim \varnothing, f(x)<0$ when $\varnothing \succ\{x\}$ and $f(x)=-f(y)$ when $(\{x\} \succ \varnothing$ and $\varnothing \succ\{y\}$ ), which in turn corresponds to a fully specified self-reflecting signed order where $g \sim_{1} b^{*} \succ_{1} n \sim_{1} n^{*} \succ_{1} b \sim_{1} g^{*}$. Here $g, n$ and $b$ are any elements of $X$ such that $\{g\} \succ \emptyset,\{n\} \sim \emptyset$ and $\emptyset \succ\{b\}$. Starting from such a self-reflecting signed order, and by identifying $\mathbf{c}$ with $f$, a GNB is the rule that satisfies all the specifications and results of what Fishburn (1992) calls "Model 2".

However, the WGNB class of rules does not enable self-reflecting signed-orders to be completely induced because the relative values of $\alpha$ and $\beta$ are unspecified. In particular, merely knowing that a rule is in the WGNB class does not enable $g$ to be compared with $b^{*}$ or $b$ with $g^{*}$ by means of $\succcurlyeq_{1}$, hence it is not possible to perform the extension analysis proposed in Fishburn (1992).

In sum, Fishburn (1992) model and ours share an interest in additive rankings of subsets that might contain good, neutral and bad elements. However there are key methodological and interpretative differences between the two approaches.

First of all, Fishburn (1992) model is developed on the basis of two important assumptions: the existence of a primitive self-reflecting signed order over $X \cup X^{*}$, and the proposal of an additive rule as a natural way to compare sets on the basis of a certain numerical function defined on $X$. This is merely further evidence of the plausibility

\footnotetext{
${ }_{8}$ Since preserving functions $f$ are not unique, the previous result does not provide a complete classification between good, indifferent and bad sets.

9 This condition is less immediate than the one used in Theorem 1, but shares the same tallying nature. We believe that a detailed description of it goes beyond the scope of a generic comparison between the two models.
} 
of additive rules, which justifies the interest of researching the axiomatic conditions needed for them to arise. Our work follows this axiomatic approach in the case of a specific subfamily of such additive rules. Moreover, the axiomatic characterization that we develop is not made as an "extension" analysis because there is no primitive relation defined over the single elements the information of which can be extended. Our primitive materials are certain conditions that the relation among sets, $\succcurlyeq$, should satisfy.

Moreover, the reason why we characterize a particular subfamily of additive rules where only certain values of $\mathbf{c}$ are feasible is that our model aims to be applicable to decision problems where simple categorization makes sense. This is clearly captured by our axioms and constitutes a central concern for us. Fishburn (1992) purpose is not to present a model of categorization: although his model certainly features a classification into good, indifferent and bad elements, there is room for as much discrimination between degrees of goodness or badness as may be desired.

Open Access This article is distributed under the terms of the Creative Commons Attribution License which permits any use, distribution and reproduction in any medium, provided the original author(s) and source are credited.

\section{References}

Alcantud JCR, Arlegi R (2008) A characterization of additively representable rankings of sets. Theory Dec 64(2-3):147-171

Allport GW (1954) The nature of prejudice. Addison Wesley, Reading

Arlegi R (2007) Sequentially consistent rules of choice under complete uncertainty. J Econ Theory 135(1):131-143

Banerjee S, Konishi T, Sönmez T (2001) Core in a simple coalition formation game. Soc Choice Welf 18(1): $135-153$

Barberà S, Barrett CR, Pattanaik P (1984) On some axioms for ranking sets of alternatives. J Econ Theory 33:301-308

Barberà S, Bossert W, Pattanaik P (2004) Ranking sets of objects. In: Barberà S, Hammond P, Seidl C (eds) Handbook of utility theory, vol 2. Kluwer Academic Publishers, Dordrecht, pp 893-977

Barberà S, Maschler M, Shalev J (2001) Voting for voters: a model of electoral evolution. Games Econ Behav 37:40-78

Barberà S, Sonnenschein H, Zhou L (1991) Voting by committees. Econometrica 59:595-609

Berga D, Bergantiños G, Massò J, Neme A (2004) Stability and voting by committees with exit. Soc Choice Welf 23:229-247

Bogomolnaia A, Jackson M (2002) The stability of hedonic coalition structures. Games Econ Behav 38:201-230

Bogomolnaia A, Moulin H, Stong R (2005) Collective choice under dichotomous preferences. J Econ Theory $122: 165-184$

Bogomolnaia A, Moulin H (2004) Random matching under dichotomous preferences. Econometrica 72(1):257-279

Bossert W, Pattanaik P, Xu Y (2000) Choice under complete uncertainty: axiomatic characterizations of some decision rules. Econ Theory 16:295-312

Brams S, Fishburn PC (2002) Voting procedures. In: Arrow KJ, Sen AK, Suzumura K (eds) Handbook of social choice and welfare, vol 1. Elsevier Science, Amsterdam, pp 173-236

Brams S, Fishburn PC (1978) Approval voting. Am Polit Sci Rev 72(3):831-847

Burani N, Zwicker WS (2003) Coalition formation games with separable preferences. Math Soc Sci 45:2752

De Finetti B (1931) Sul significato soggettivo della probabilità. Fundam Math 17:298-329 
Dimitrov D, Borm P, Hendrickx R, Sung SC (2006) Simple priorities and core stability in hedonic games. Soc Choice Welf 26:421-433

Dimitrov D, Sung SC, Xu Y (2007) Procedural group identification. Math Soc Sci 54(2):137-146

Fishburn PC (1992) Signed orders and power set extensions. J Econ Theory 56:1-19

Gale D, Shapley LS (1962) College admisions and the stability of marriage. Am Math Mon 69(1):9-15

Gärdenfors P (1976) Manipulation of social choice functions. J Econ Theory 13:217-228

Jones P, Sugden R (1982) Evaluating choice. Int Rev Law Econ 2:47-65

Ju BG (2003) A characterization of strategy-proof voting rules for separable weak orderings. Soc Choice Welf 21:469-499

Ju BG (2005) An efficiency characterization of plurality social choice on simple preference domains. Econ Theory 26:115-128

Kelly JS (1977) Strategy-proofness and social choice functions without single-valuedness. Econometrica 45:439-446

Kraft CH, Pratt JW, Seidenberg A (1959) Intuitive probability on finite sets. Ann Math Statist 30:408-419

Pattanaik PK (1978) Strategy and group choice. North-Holland, Amsterdam

Pattanaik PK, Xu Y (1990) On ranking opportunity sets in terms of freedom of choice. Rech Econ Louv 56:383-390

Roth AE, Sotomayor M (1990) Two-sided matching: a study in game-theoretic modeling and analysis. Cambridge University Press, Cambridge

Romero-Medina A (2001) More on preferences and freedom. Soc Choice Welf 18:179-191

Samet D, Schmeidler D (2003) Between liberalism and democracy. J Econ Theory 110:213-233

Scott D (1964) Measurement structures and linear inequalities. J Math Psychol 1:233-247

Sugden R (1998) The metric of opportunity. Econ Philos 14:307-337 